\title{
Prognostic impact of para-aortic lymph node metastases in non-pancreatic periampullary cancer
}

\author{
Sebastian Hempel ${ }^{1} \mathbb{D}$, Florian Oehme ${ }^{1}$, Benjamin Müssle ${ }^{1}$, Daniela E. Aust ${ }^{2}$, Marius Distler ${ }^{1}$, Hans-Detlev Saeger ${ }^{1}$, \\ Jürgen Weitz ${ }^{1}$ and Thilo Welsch ${ }^{*}$
}

\begin{abstract}
Background: Resection of the para-aortic lymph node (PALN) group Ln16b1 during pancreatoduodenectomy remains controversial because PALN metastases are associated with a worse prognosis in pancreatic cancer patients. The present study aimed to analyze the impact of PALN metastases on outcome after non-pancreatic periampullary cancer resection.

Methods: One hundred sixty-four patients with non-pancreatic periampullary cancer who underwent curative pancreatoduodenectomy or total pancreatectomy between 2005 and 2016 were retrospectively investigated. The data were supplemented with a systematic literature review on this topic.

Results: In 67 cases, the PALNs were clearly assigned and could be histopathologically analyzed. In 10.4\% of cases (7/67), tumor-infiltrated PALNs (PALN+) were found. Metastatic PALN+ stage was associated with increased tumor size $(P=0.03)$ and a positive nodal stage $(P<0.001)$. The median overall survival $(O S)$ of patients with metastatic PALN and non-metastatic PALN (PALN-) was 24.8 and 29.5 months, respectively. There was no significant difference in the OS of PALN + and pN1 PALN patients $(P=0.834)$. Patients who underwent palliative surgical treatment $(n=$ 20) had a lower median OS of 13.6 (95\% confidence interval 2.7-24.5) months. Including the systematic literature review, only 23 cases with PALN+ status and associated OS could be identified; the average survival was 19.8 months.

Conclusion: PALN metastasis reflects advanced tumor growth and lymph node spread; however, it did not limit overall survival in single-center series. The available evidence of the prognostic impact of PALN metastasis is scarce and a recommendation against resection in these cases cannot be given.
\end{abstract}

Keywords: Non-pancreatic periampullary cancer, Para-aortic lymph nodes, Pancreatoduodenectomy, Survival

\section{Introduction}

The treatment of choice for patients affected by periampullary cancer is a pancreaticoduodenectomy (PD) [1]. The spread of cancer to regional lymph nodes is an important prognostic factor after resection, independent of cancer histology [2]. Therefore, lymphadenectomy is considered a critical step of PD for cancer. While there is consensus about the major steps and extent of lymphadenectomy during $\mathrm{PD}$, no strong recommendation is

\footnotetext{
* Correspondence: thilo.welsch@uniklinikum-dresden.de

1 Department of Visceral, Thoracic and Vascular Surgery, University Hospital

Carl Gustav Carus, Technische Universität Dresden, Dresden, Germany

Full list of author information is available at the end of the article
}

given for routine resection of para-aortic lymph node (PALN) station Ln16b1 [3]. These lymph nodes are located dorsal to the pancreas and do not belong to the regional lymph node stations. However, the Ln16b1 station is an important node in major lymphatic drainage [3]. Metastatic spread to this lymph node station is therefore classified as pM1 stage. Several studies-including our own recent work-concluded that tumor-bearing PALNs of pancreatic ductal adenocarcinoma (PDAC) were a predictor for poor overall survival [4-7]. The majority of available data investigates the impact of PALNs in patients with PDAC, but without non-pancreatic periampullary

(c) The Author(s). 2020 Open Access This article is distributed under the terms of the Creative Commons Attribution 4.0 International License (http://creativecommons.org/licenses/by/4.0/), which permits unrestricted use, distribution, and 
cancer. However, histopathologic phenotype seems to be an important and relevant prognostic factor affecting survival in patients with periampullary tumors [8]. The biological behavior of PALN metastasis of intestinal-type non-pancreatic periampullary cancer probably differs from that of the pancreaticobiliary types, such as PDAC, and has a better prognosis. There is little data on the outcome of PALN metastasis in non-pancreatic periampullary cancer. Thus, the primary aim of the present study was to investigate the subgroup of non-pancreatic periampullary cancer resections in our center with respect to PALN metastases and provide a systematic literature review of this topic.

\section{Methods}

\section{Study design and patients}

All patients who underwent partial pylorus-preserving PD (PPPD), classic PD (cPD), or total pancreatectomy (TP) for non-pancreatic periampullary cancer between January 2005 and December 2016 at the Department of Visceral, Thoracic and Vascular Surgery, University Hospital Carl Gustav Carus, Technische Universität Dresden, Germany were identified from a pancreatic database and retrospectively analyzed. Resection of lymph node Ln16b1 (limited PALN resection) was performed routinely. Nevertheless, the resected lymph node specimens could not be clearly assigned to PALN station Ln16b1 in all cases during the pathological work-up. This was because PALNs were not unequivocally labeled during every operation, thereby making it impossible to clearly differentiate them from peripancreatic lymph nodes in some cases. For the present study, only cases with clearly labeled PALNs were considered. These cases were divided into two groups: patients with a PALNpositive (PALN+) status (histopathological tumor invasion of at least one PALN) and patients without PALN metastasis (PALN-). The latter group was further stratified based on nodal status ( $\mathrm{pNO}$ or $\mathrm{pN}+$ ). An additional comparative survival analysis was computed by taking into account all patients who underwent palliative surgery for metastatic or unresectable stage within the same observation time.

To evaluate tumor recurrence, follow-up data were collected during regular examinations in our outpatient clinic, as well as through phone calls or interviews with primary physicians. The clinical examination, an elevated carbohydrate antigen 19-9 (CA 19-9) level or imaging modalities (e.g., sonography, computed tomography (CT) or magnetic resonance imaging (MRI) scans) were used to detect tumor progression. The experimental protocol of the study was approved by the local ethics committee of the TU Dresden (decision number EK70022017).

\section{Operative technique}

The principal operative techniques of PPPD and cPD have recently been described [9]. With respect to the lymphadenectomy, the PALNs (Ln16b1) were dissected dorsal to the pancreas during an extended Kocher maneuver. The PALNs were resected between the vena cava and aorta, including the ventral aspect of these vessels, beginning from the left renal vein to the superior margin of the inferior mesenteric artery. This PALN subgroup was routinely resected because it was included within the primary resection plane and the dissection was helpful for clear visualization of the superior mesenteric artery origin; other PALN stations such as Ln16b2 were not resected.

\section{Pathological assessment}

After macroscopic examination, the resected para-aortic lymph node (PALN) specimens were histologically analyzed using hematoxylin and eosin slides without serial sectioning or immunohistochemical analyses for single tumor cells. Once tumor infiltration with desmoplastic reaction was detected, PALN metastasis was diagnosed.

\section{Systematic review}

The systematic review was performed according to the Preferred Reporting Items for Systematic Reviews and Meta-Analysis (PRISMA) guidelines [10]. All published articles available in the MEDLINE, Embase, Web of

Table 1 Patient and operative characteristics

\begin{tabular}{llll}
\hline Variable & PALN + & PALN- & $P$ \\
\hline Patients [n] & 7 & 60 & \\
Median age [years] (IQR) & $67(64-73)$ & $71(63-75)$ & 0.798 \\
Male sex [n/(\%)] & $2(29)$ & $35(58)$ & 0.228 \\
Diabetes [n/(\%)] & $2(29)$ & $8(13)$ & 0.278 \\
Weight loss [n/(\%)] & $5(71)$ & $27(45)$ & 0.245 \\
Jaundice [n/(\%)] & $5(71)$ & $32(53)$ & 0.446 \\
Median CA 19-9 & $166(28-530)$ & $46(15-139)$ & 0.583 \\
[U/ml] (IQR) & & & \\
Operation [n/(\%)] & & $50(83)$ & 0.582 \\
$\quad$ PPPD & $7(100)$ & $9(15)$ & 0.581 \\
$\quad$ CPD & 0 & $1(2)$ & 1.000 \\
$\quad$ TP & 0 & 0 & \\
Neoadj. therapy [n/(\%)] & 0 & $11(18)$ & 1.000 \\
Portal vein resection [n/(\%)] & $1(14)$ & $363(298-439)$ & 0.573 \\
Median operative time & $375(313-473)$ & $17(28)$ & 0.193 \\
[min] (IQR) & & $4(57)$ &
\end{tabular}

Abbreviations: PPPD: Pylorus-preserving pancreaticoduodenectomy; CPD: Classic pancreatoduodenectomy; TP: Total pancreatectomy; neoadj:. Neoadjuvant; IQR: Interquartile range 
Science, and Cochrane Library databases up to August 18, 2019 were screened. A search algorithm consisting of the corresponding $\mathrm{MeSH}$ terms was developed and adapted for the respective database. The search algorithm for the MEDLINE database (PubMed) is given in Table 5. The reference lists of all included articles were manually cross-checked in order to identify additional studies.

Two independent investigators (Sebastian Hempel and Benjamin Müssle) evaluated each article for inclusion. After removing the duplicates, all case reports and studies investigating inappropriate tumor entity were excluded. Only studies providing data on the results of overall survival after resection of non-pancreatic

Table 2 Histopathological tumor characteristics

\begin{tabular}{|c|c|c|c|}
\hline Variable & PALN+ & PALN- & $P$ \\
\hline Patients $[n]$ & 7 & 60 & - \\
\hline \multicolumn{4}{|l|}{ Tumor histology } \\
\hline Distal bile duct carcinoma & $5(71)$ & $30(50)$ & 0.430 \\
\hline Ampullary carcinoma & $2(29)$ & $25(40)$ & 0.692 \\
\hline Carcinoma of duodenum & 0 & $5(8)$ & 1.000 \\
\hline \multicolumn{4}{|l|}{ Tumor grade $[n /(\%)]$} \\
\hline G1/G2 & $2(29)$ & $24(40)$ & 0.697 \\
\hline G3/G4 & $5(71)$ & $35(58)$ & 0.692 \\
\hline Gx & 0 & $1(2)$ & 1.000 \\
\hline \multicolumn{4}{|l|}{ pT } \\
\hline $\mathrm{T} 1 / \mathrm{T} 2$ & $2(29)$ & $23(38)$ & 0.704 \\
\hline $\mathrm{T} 3 / \mathrm{T} 4$ & $5(71)$ & $37(62)$ & \\
\hline \multicolumn{4}{|l|}{$\mathrm{pN}$} \\
\hline No & 0 & $29(48)$ & 0.016 \\
\hline $\mathrm{N} 1$ & $7(100)$ & $31(52)$ & \\
\hline \multicolumn{4}{|l|}{$\mathrm{pM}$} \\
\hline MO & 0 & $60(100)$ & \\
\hline M1 & $7(100)$ & & 0.001 \\
\hline \multicolumn{4}{|l|}{ Resection status } \\
\hline RO & $7(100)$ & $55(92)$ & 1.000 \\
\hline R1 & 0 & $5(8)$ & \\
\hline Tumor size [mm] (IQR) & $30(22-35)$ & $20(15-30)$ & 0.282 \\
\hline Median number of resected LN (IQR) & $21(18-25)$ & $20(13-25)$ & 0.857 \\
\hline Median number of resected PALN (IQR) & $5(1-6)$ & $4(2-6)$ & 0.556 \\
\hline \multicolumn{4}{|l|}{ LN ratio } \\
\hline$>0.2$ & $5(71)$ & $8(13)$ & 0.002 \\
\hline$\leq 0.2$ & $2(29)$ & $52(87)$ & \\
\hline \multicolumn{4}{|l|}{ PALN ratio } \\
\hline$>0.2$ & $6(86)$ & 0 & 0.001 \\
\hline$\leq 0.2$ & $1(14)$ & $60(100)$ & \\
\hline
\end{tabular}

Abbreviations: IQR: Interquartile range; $L N$ : Lymph node $p$ values $<0.05$ are in boldface periampullary cancer with and without PALN metastasis were considered.

\section{Statistical analysis}

The IBM SPSS 25 (SPSS Statistics v25, IBM Corporation, Armonk, New York) software package was used for statistical analysis and data plots. A value of $P<0.05$ was considered statistically significant. Categorical and quantitative variables were analyzed using Fisher's exact test and the unpaired $t$-test, respectively. All quantitative variables were expressed as median with interquartile range (IQR). Cox proportional hazards models were used to compute uni- and multivariate survival analyses. Patient age $>70$ years, $\mathrm{T}$ and $\mathrm{N}$ stage, tumor grade, resection stage, neo- and adjuvant treatment, and PALN status were considered for univariate analysis. The quotient between tumor-infiltrated lymph nodes and resected lymph nodes was defined as the lymph node ratio (LNR) and stratified in LNR $<0.2$ and $\geq 0.2$. Variables found to be significant in univariate analysis were further used for multivariate testing. The number of positive and resected lymph nodes, tumor size, preoperative CA 19-9, and progression-free survival (PFS) time were considered for a multiple linear regression model. The overall survival and progression-free survival curves were determined using the Kaplan-Meier method. Differences between the survival curves were identified using the log-rank test. Overall survival (OS) was calculated as the period between the index operation until the date of death or the time of last contact (censored). Similarly, PFS was defined as the time to last follow-up contact without the progression of the tumor. The

Table 3 Operative morbidity and mortality

\begin{tabular}{llll}
\hline Variable & PALN+ & PALN- & $P$ \\
\hline Patients $[n]$ & 7 & 60 & \\
Morbidity $[n /(\%)]$ & $6(86)$ & $39(60)$ & 0.411 \\
$\quad$ Diarrhea $[n /(\%)]$ & 0 & 0 & \\
Lymph fistula [n/(\%)] & 0 & $3(5)$ & 1.000 \\
POPF [n/(\%)] & $1(14)$ & $21(35)$ & 0.411 \\
PPH $[n /(\%)]$ & 0 & $9(15)$ & 0.581 \\
DGE $[n /(\%)]$ & $1(14)$ & $25(42)$ & 0.233 \\
SSI $[n /(\%)]$ & $2(29)$ & $14(23)$ & 1.000 \\
Abscess [n/(\%)] & $2(29)$ & $16(26)$ & 1.000 \\
Thromboembolism $[n /(\%)]$ & $1(14)$ & $4(7)$ & 0.434 \\
Mortality & & & \\
30d $[n /(\%)]$ & 0 & $3(5)$ & 1.000 \\
60d $[n /(\%)]$ & 0 & $3(5)$ & 1.000 \\
\hline Abbrevatons POPF: Postoper
\end{tabular}

Abbreviations: POPF: Postoperative pancreatic fistula B/C (classified according to Bassi); PPH: Postpancreatectomy hemorrhage; DGE: Delayed gastric emptying; SSI: Surgical site infection 
period from surgery to last patient contact or death of the patient was defined as the follow-up time.

\section{Results}

Patient cohort and histopathological tumor characteristics One hundred sixty-four patients underwent pancreatoduodenectomy (PD) or total pancreatectomy (TP) for non-pancreatic periampullary cancer within the study period. In 67 cases (41\%), the resected PALNs were separately labeled and histopathologically analyzed, whereas the resected lymph nodes could not be retrospectively assigned to the PALN compartment in the remaining cases. Tumor-infiltrated PALNs (PALN+) were proved in $10.4 \%$ of cases $(7 / 67)$. In most cases, a pylorus-preserving pancreatoduodenectomy (PPPD) was performed (84\%). No significant differences in the majority of standard patient and surgical characteristics were found between PALN+ and PALN- patients (Table 1). However, the PALN+ patients tended to have higher preoperative serum CA 19-9 levels. Furthermore, metastatic PALNs were always associated with regional lymph node infiltration. The significantly higher rate of nodal-positive status (pN1) and higher lymph node ratio reflect advanced lymphovascular spreading (Table 2). In addition, PALN metastases were significantly correlated with the number of metastatic regional lymph nodes $(P<$ $0.001)$ and tumor size $(P=0.03)$ in a multiple linear regression model. In the most of PALN+ cases, only one PALN was tumor infiltrated (4 of 7 cases; range of metastatic PALN: 1-3). Histopathological reporting of the tumor subtype was available in 6 of the 7 PALN cases: 3 were intestinal, 2 were pancreaticobiliary, and 1 was of a mixed subtype.

No significant differences with regard to postoperative morbidity were detected in either subgroup (Table 3). However, the rate of postoperative pancreatic fistula (POPF) and delayed gastric emptying (DGE) was slightly higher in PALN- patients than in PALN+ patients $(36 \%$ vs. $14 \%, 42 \%$ vs. $14 \%)$. The overall 30 -day mortality was $4.4 \%(3 / 67)$.

\section{Survival analysis}

The survival analysis was calculated using the KaplanMeier method with log-rank test. The median OS of PALN+ and PALN- patients was 24.8 (95\% confidence interval [CI] 14.6-35.0) months and 29.5 (95\% CI 8.250.8 months, respectively. Figure 1a shows the OS curves of the two subgroups. The median PFS in the PALN+ group was 8.3 (95\% CI 5.5-11.1) months, whereas in the PALN- group it was 23.7 (95\% CI 051.5). However, no statistical significance was calculated $(P=0.29)$. The curves of PFS of the two subgroups (PALN+ vs. PALN-) are shown in Fig. 2a. On univariate analysis, no significant correlation between PALN status and overall survival was found $(P=0.163)$. Only two significant factors affecting overall survival rates were identified on multivariate analysis (Table 4): nodal status $(P<0.01)$ and failure to receive adjuvant treatment $(P<0.01)$.

In order to compare PALN with regional lymph node metastases, we generated two subgroups within the nonmetastatic PALN cohort according to their nodal stage into 29 cases without regional lymph node metastasis
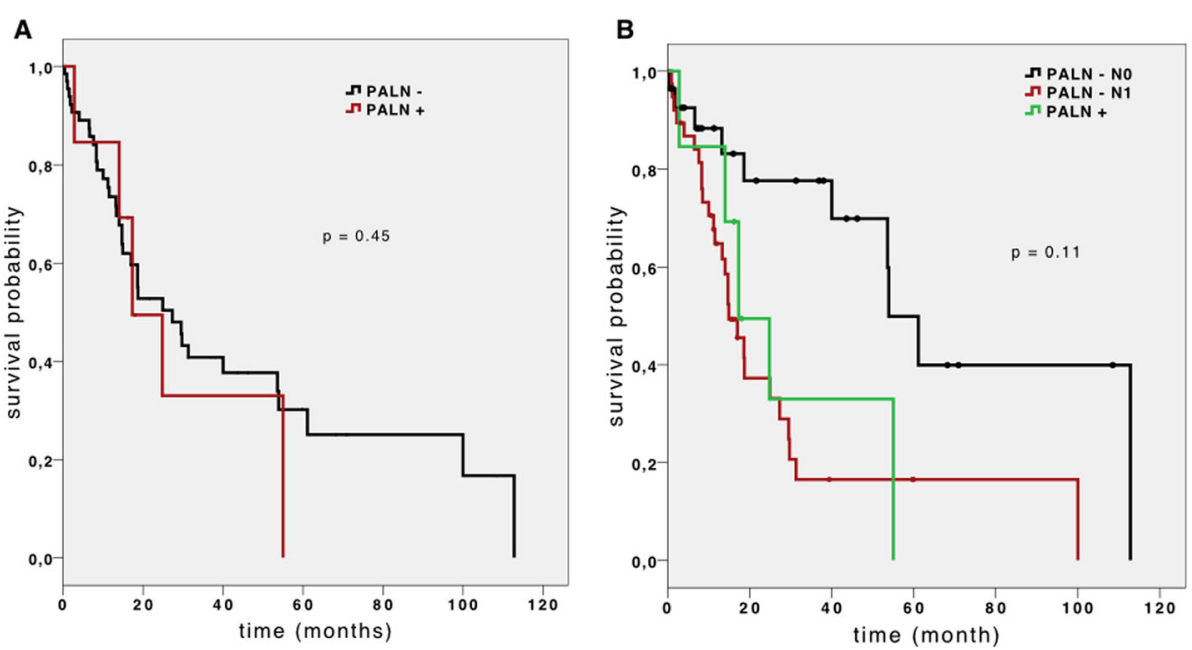

Fig. 1 Overall survival of patients with para-aortic lymph node resection. a Overall survival of patients with para-aortic lymph node (PALN) resection $(n=67)$. Patient subgroups with PALN metastasis (PALN,$+ n=7)$ and without PALN metastasis (PALN-, $n=60)$ were plotted. b Overall survival of patients with PALN resection separated into PALN $+(n=7)$, PALN - pNO $(n=29)$, and PALN- pN1 $(n=31)$ subgroups according to the regional lymph node status 
and 31 nodal-positive (pN1) cases. In the $\mathrm{pN} 0$ subgroup, we noticed a significantly longer $(P=0.011)$ median overall survival (53.9 months, 95\% CI 43.3-64.4) than that of the $\mathrm{pN} 1$ subgroup (OS 17.0 months, 95\% CI 11.7-22.2) or PALN+ cohort. Figure $1 \mathrm{~b}$ shows the OS curves of these three subgroups. On the other hand, there was no significant difference in median OS of the PALN- pN1 and PALN+ $(P=0.834)$ subgroups. Furthermore, the PALN- pN0 subgroup also had a significantly longer PFS $(P=0.033)$. In Fig. $2 b$, the PFS curves of these three subgroups are shown.

We further analyzed the survival probability of PALN+ patients and all patients treated palliatively within the study period. Twenty patients who underwent surgical exploration or palliative bypass surgery for unresectable or metastatic, non-pancreatic periampullary cancer were identified. The median OS of these 20 patients was 13.6 (95\% CI 2.7-24.5) months and significantly shorter than that of the PALN+ subgroup $(P=0.03)$.

\section{Systematic literature review}

Due to the limited cohort size of patients with nonpancreatic periampullary cancers and PALN data, we conducted a systematic electronic literature search (Table 5).
A total of 87 abstracts were identified. After a detailed assessment according to the inclusion criteria, only 2 studies could be considered for further data extraction (Fig. 3). These 2 studies combined with our present data provided data on 23 patients with PALN metastases after resection of nonperiampullary cancers. In 2004, Yoshida et al. reported on 6 of 36 PALN-positive patients, with a median overall survival of 19 months [11]. In 2016, Hafeez Bhatti et al. reported on 10 of 40 patients with involved PALN and a median overall survival of 17.5 months [12] (Table 6). The mean survival of the 23 patients with PALN metastases was 19.8 months.

\section{Discussion}

Regarding on the management of PALN, no consensus has been reached so far [3]. Extended lymphadenectomy or PALN resection during oncological PD is still an ongoing discussion and area of controversy. The present study demonstrates that approximately $10 \%$ of nonpancreatic periampullary cancer cases harbor PALN metastases at the time of resection. Previous studies have reported comparable sample sizes (Table 7) with a higher incidence of PALN metastasis (17-25\%) [11, 12]. In the study by Connor et al., the incidence of PALN

\section{A}

B
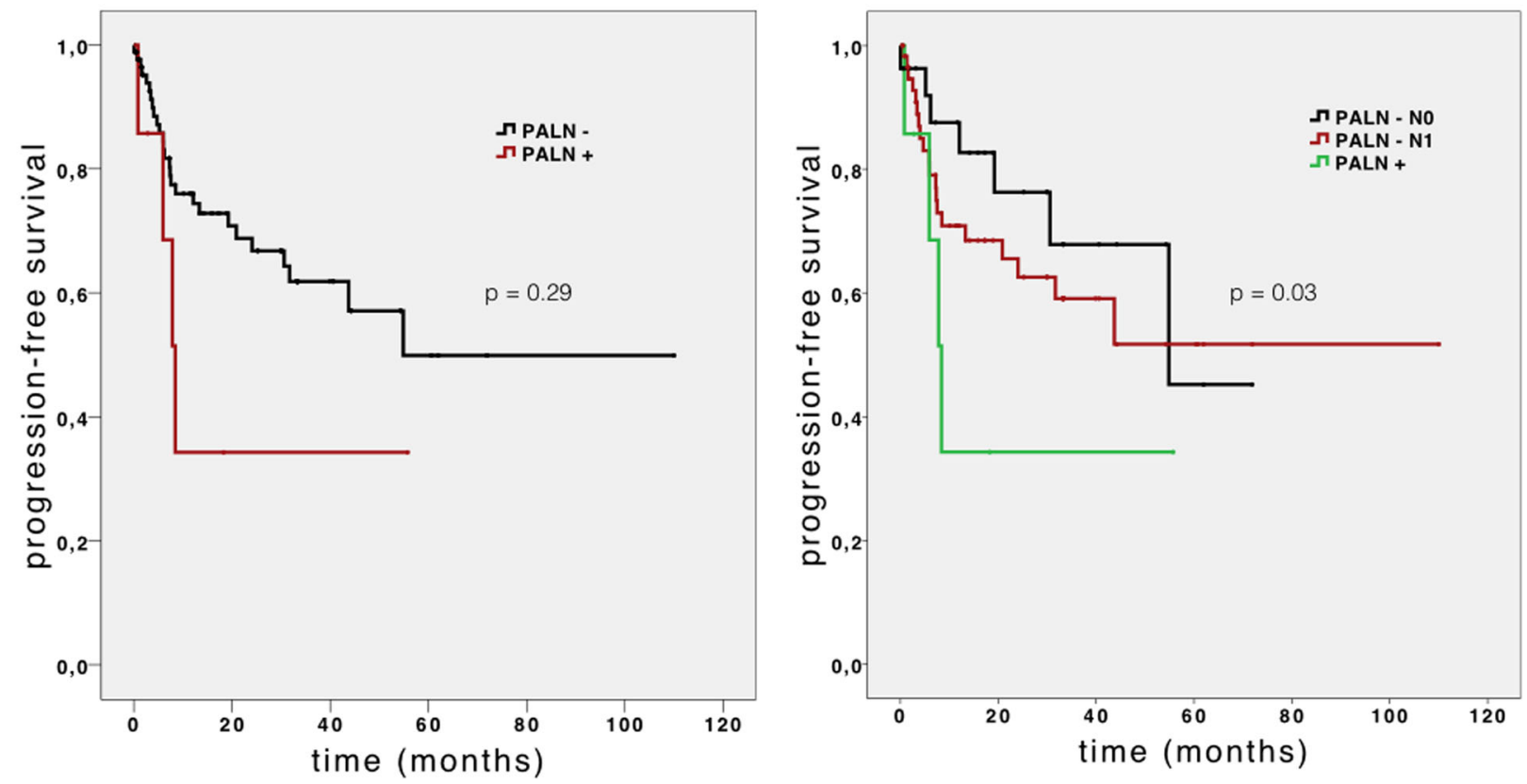

Fig. 2 Progression-free survival of patients with para-aortic lymph node resection. a Progression-free survival of patients with para-aortic lymph node (PALN) resection $(n=67)$. Patient subgroups with PALN metastasis (PALN,$+ n=7)$ and without PALN metastasis $($ PALN-, $n=60)$ were plotted. b Progression-free survival of patients with PALN resection separated into PALN+ $(n=7), \mathrm{PALN}-\mathrm{pNO}(n=29)$, and PALN- pN1 $(n=31)$ subgroups according to the regional lymph node status 
Table 4 Univariate and multivariate analysis

\begin{tabular}{lllll}
\hline Variable & Univariate analysis & \multicolumn{3}{l}{ Multivariate analysis } \\
\cline { 3 - 5 } & $P$ & OR & $95 \% \mathrm{Cl}$ & $P$ \\
\hline Age $>$ 70 years & 0.07 & 1.138 & $0.350-3.701$ & 0.83 \\
pT-stage & 0.08 & 1.804 & $0.707-4.606$ & 0.217 \\
pN-stage & $\mathbf{0 . 0 2}$ & 6.124 & $1.377-27.239$ & $<\mathbf{0 . 0 1}$ \\
Grading & 1 & 1.255 & $0.414-3.806$ & 0.69 \\
Resection status & 0.64 & 0.459 & $0.034-6.177$ & 0.56 \\
Adjuvant therapy & 0.13 & 0.184 & $0.04-0.860$ & $<\mathbf{0 . 0 1}$ \\
PALN status & 0.163 & 0.756 & $0.099-5.804$ & 0.79 \\
\hline
\end{tabular}

Abbreviations: Cl: Confidence interval; IQR: Interquartile range; OR: Odds ratio LN: Lymph node

$p$ values $<0.05$ are in boldface

metastases in non-pancreatic periampullary cancer (only distal bile duct carcinoma considered) was significantly higher at $55 \%$ [13].

Yoshida et al., reported a median overall survival of 19 months in cases of PALN metastasis; however, only cases of distal bile duct involvement were investigated [11]. In 2016, Hafeez Bhatti et al. demonstrated comparable survival data between PALN+ (17.5 months) and PALN- subgroups (17 months). In our analysis, the patients with and without PALN metastases survived 24.8 and 29.5 months, respectively. These findings indicate that PALN+ and PALN- status may lead to similar survival after resection. However, there seems to be a difference in median overall survival and the frequency of PALN metastasis in non-

Table 5 Search strategy and results for the systematic literature review as used for MEDLINE

\begin{tabular}{|c|c|c|}
\hline Search & Query & $\begin{array}{l}\text { Items } \\
\text { found }\end{array}$ \\
\hline \multicolumn{3}{|c|}{ Population } \\
\hline$\# 1$ & $\begin{array}{l}\text { Distal AND (bile duct cancer OR } \\
\text { cholangiocarcinoma) OR "Periampullary cancer" } \\
\text { OR "peri-ampullary" OR adenocarcinoma AND" } \\
\text { (bile duct OR ampulla) OR "ampullary cancer" }\end{array}$ & 14727 \\
\hline \multicolumn{3}{|c|}{ Intervention } \\
\hline$\# 2$ & $\begin{array}{l}\text { Pancreatoduodenectomy OR } \\
\text { pancreaticoduodenectomy OR } \\
\text { duodenopancreatectomy OR pancreatectomy } \\
\text { OR Whipple OR "Pancreatic head resection" OR } \\
\text { "surgical resection" OR resection }\end{array}$ & 291546 \\
\hline \multicolumn{3}{|c|}{ End points } \\
\hline \#3 & $\begin{array}{l}\text { (Interaortocaval OR para-aortal OR para-aortic) } \\
\text { AND ("Iymph nodes" OR metastas* OR } \\
\text { lymphadenectomy) OR PALN }\end{array}$ & 3528 \\
\hline \multicolumn{3}{|c|}{ Synthesis } \\
\hline \#4 & \#1 AND \#2 AND \#3 & 40 \\
\hline
\end{tabular}

pancreatic periampullary cancer between Asian and European populations [11, 12].

The relevance of the extent of lymphadenectomy in resected periampullary cancer has been described by some previous studies [15-17]. In general, the lymph node ratio rather than lymph node status was identified as a prognostic factor. Recently, Liu et al. published a definition of an extended minimum level of at least 16 lymph nodes in non-pancreatic periampullary cancer resections [18].

A comparison of the median survival duration of patients with palliative surgery (13.6 months) or PALN+ resection (24.8 months) showed that patients in the latter subgroup demonstrated a longer survival of approximately 10 months. Thus, PALN resection (Ln16b1) in cases of non-pancreatic periampullary cancer may provide an advantage for patients.

A recent study reported the impact of the histopathologic phenotype of periampullary cancer on survival and response to therapy [8]. The findings highlight the clear need to assess tumor biology and growth, treatment regimen and the prognosis of peripancreatic tumors with respect to genetic, molecular or histologic subtypes in the future. Based on the findings of Williams et al., the intestinal phenotype is associated with a better outcome than the pancreatobiliary phenotype. In the present study, the following subtypes were found in 6 of the 7 PALN cases: 3 intestinal, 2 pancreaticobiliary, and 1 mixed type. Therefore, we could not prove the influence of histopathologic phenotype on PALN metastasis.

A recent meta-analysis explored the prognostic value of lymph node metastases in pancreatic and periampullary cancer [19]. Most of the included studies focused on the impact of PALN metastasis; however, isolated survival data in cases of PALN metastases detected during surgical resection for non-pancreatic periampullary cancer were not provided.

The results and conclusions of the present analysis are significantly limited by the small number of available data sets (7 patients with PALN metastases). PALN resection was routinely performed at our institution; however, a definite allocation of the resected lymph nodes to the Ln16b1 station (PALN) was only possible in 67 of the 164 cases. This was due to the fact that PALNs were not routinely labeled at the time of resection. Nevertheless, the tumor biology of intestinal-type tumors in the periampullary region is less aggressive than pancreatic cancer. In addition, the little available data must be analyzed to determine the optimal operative treatment of this cancer subtype. All previous studies reporting on periampullary cancers and PALN metastases investigated a similar number of cases with comparable survival data [11, 
85 records identified

through database searching:

40 PubMed

12 Embase

$30 \mathrm{Web}$ of Science

3 Cochrane

2 additional records identified through other sources

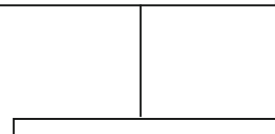

56 records after duplicates removed

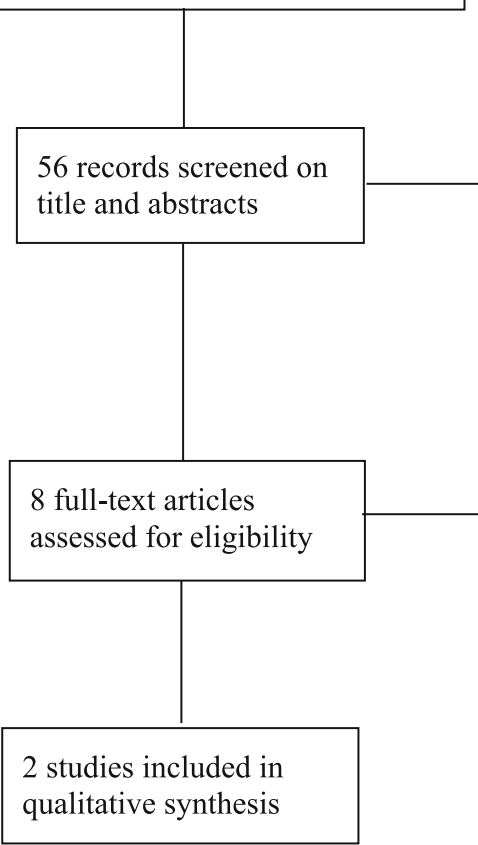

48 records excluded:

-other tumor entity: 28

-case reports: 9

-trial protocol: 1

-congress letter: 1

-no PALN data: 9

6 full-text articles excluded:

-no original data available: 1

-no OS data for non-pancreatic

periampullary cancer: 4

-no PALN analysis performed: 1

Fig. 3 PRISMA flow diagram of the systematic literature search

12]. Contrary to other studies on PALN resection, the present study explicitly analyzed cases of nonpancreatic periampullary cancer.

\section{Conclusion}

The present study indicates that patients with nonpancreatic periampullary cancers and PALN metastases (pM1-LYM) may have a prognosis similar to that of patients without PALN metastases, however, this observation is based on very few cases. This is in contrast to pancreatic cancer patients, who have a worse survival in cases of PALN metastases [4-7]. Although tumor infiltration of PALNs is an indicator of advanced tumor growth and regional lymph node metastasis, patients with non-pancreatic periampullary cancer and suspected or proven PALN metastases may benefit from aggressive tumor resection. This management approach could be further supported by an improved current neo-

Table 6 Results of the systematic literature review

\begin{tabular}{|c|c|c|c|c|c|c|}
\hline Reference & Year & Study design & Analyzed cases ( $n$ ) & PALN+ cases $(n)$ & $\begin{array}{l}\text { Median } \\
\text { PALN+ }\end{array}$ & \\
\hline Yoshida et al. .[11] & 2004 & Retrospective & 36 & 6 & 19.0 & $\mathrm{n} / \mathrm{a}$ \\
\hline Hafeez Bhatti et al .[12] & 2016 & Retrospective & 40 & 10 & 17.5 & 17.0 \\
\hline Present study & 2019 & Retrospective & 67 & 7 & 24.8 & 29.5 \\
\hline
\end{tabular}

Abbreviations: PALN: Para-aortic lymph node; OS: Overall survival 
Table 7 Incidence of PALN metastases in non-pancreatic periampullary cancer

\begin{tabular}{|c|c|c|c|c|c|c|}
\hline Reference & Year & Study design & Analyzed cases ( $n$ ) & PALN+ cases $[n /(\%)]$ & Population & Histology \\
\hline Yoshida et al .[11] & 2004 & Retrospective & 36 & $6(17)$ & $A$ & DBC \\
\hline Hafeez Bhatti et al .[12] & 2016 & Retrospective & 40 & $10(25)$ & A & Non-pancreatic \\
\hline Connor et al .[13] & 2004 & Retrospective & 11 & $6(55)$ & $E$ & DBC \\
\hline Nappo et al .[6] & 2015 & Retrospective & 49 & $3(6)$ & $E$ & $A C, D B C$ \\
\hline Murakami et al .[14] & 2011 & Retrospective & 63 & $9(14)$ & $A$ & $A C, D B C$ \\
\hline Present study & 2019 & Retrospective & 67 & $7(10)$ & $E$ & Non-pancreatic \\
\hline
\end{tabular}

Abbreviations: A: Asian; $A C$ : Ampullary cancer; $D B C$ : Distal bile duct cancer; $E$ : European

or adjuvant chemotherapeutic regimen, e.g., gemcitabine/capecitabine or Folfirinox. In the future, subtype characterization of the tumor entities in the periampullary region (e.g., intestinal and pancreaticobiliary subtypes) will probably serve as a guide for resection strategies. This study demonstrates the need for differential analysis of peripancreatic tumor entities in the future.

\section{Abbreviations}

BMI: Body mass index; CPD: Classic pancreaticoduodenectomy;

$\mathrm{CT}$ : Computed tomography; DGE: Delayed gastric emptying;

ISGPF: International Study Group for Pancreatic Fistula; LNR: Lymph node ratio; MRI: Magnetic resonance imaging; OS: Overall survival; PALNs: Paraaortic lymph nodes; PD: Pancreaticoduodenectomy; PDAC: Pancreatic ductal adenocarcinoma; PFS: Progression-free survival; POPF: Postoperative pancreatic fistula; PPH: Postpancreatectomy hemorrhage; PPPD: Pyloruspreserving pancreatoduodenectomy; SSI: Surgical site infection; TP: total pancreatectomy

\section{Acknowledgements}

Not applicable.

\section{Authors' contributions}

$\mathrm{SH}$ performed most of the data acquisition and analysis and drafted the manuscript. FO helped with the statistical analysis and reviewed the manuscript. BM and MD contributed to the data collection and reviewed the manuscript. DEA was responsible for the pathological analysis and reporting. HDS helped with the conceptual design and critically reviewed the manuscript. JW and TW designed the study, contributed to the statistical analysis and data presentation, and finalized the manuscript. All authors read and approved the final manuscript.

\section{Funding}

The present work was not supported by any third-party funding or research grants.

\section{Availability of data and materials}

The datasets that were analyzed during the current study are available from the corresponding author upon reasonable request.

\section{Ethics approval and consent to participate}

The experimental protocol of the study was approved by the local ethics committee of the TU Dresden (decision number EK70022017).

\section{Consent for publication}

Not applicable.

\section{Competing interests}

The authors declare that they have no conflicts of interest with respect to this work.

\section{Author details}

'Department of Visceral, Thoracic and Vascular Surgery, University Hospital Carl Gustav Carus, Technische Universität Dresden, Dresden, Germany.
${ }^{2}$ Institute of Pathology, University Hospital Carl Gustav Carus, TU Dresden, Dresden, Germany.

Received: 15 October 2019 Accepted: 31 December 2019

Published online: 21 January 2020

\section{References}

1. Loos M, Kleeff J, Friess H, Buchler MW. Surgical treatment of pancreatic cancer. Ann N Y Acad Sci. 2008;1138:169-80.

2. Zacharias T, Jaeck D, Oussoultzoglou E, Neuville A, Bachellier P. Impact of lymph node involvement on long-term survival after R0 pancreaticoduodenectomy for ductal adenocarcinoma of the pancreas. J Gastrointest Surg. 2007;11(3):350-6.

3. Tol JA, Gouma DJ, Bassi C, Dervenis C, Montorsi M, Adham M, et al. Definition of a standard lymphadenectomy in surgery for pancreatic ductal adenocarcinoma: a consensus statement by the International Study Group on Pancreatic Surgery (ISGPS). Surgery. 2014;156(3):591-600.

4. Schwarz L, Lupinacci RM, Svrcek M, Lesurtel M, Bubenheim M, Vuarnesson $\mathrm{H}$, et al. Para-aortic lymph node sampling in pancreatic head adenocarcinoma. Br J Surg. 2014;101(5):530-8.

5. Shimada K, Sakamoto $Y$, Sano T, Kosuge T. The role of paraaortic lymph node involvement on early recurrence and survival after macroscopic curative resection with extended lymphadenectomy for pancreatic carcinoma. Journal of the American College of Surgeons. 2006;203(3):345-52

6. Nappo G, Borzomati D, Perrone G, Valeri S, Amato M, Petitti T, et al. Incidence and prognostic impact of para-aortic lymph nodes metastases during pancreaticoduodenectomy for peri-ampullary cancer. HPB. 2015; 17(11):1001-8.

7. Hempel S, Plodeck V, Mierke F, Distler M, Aust DE, Saeger HD, et al. Paraaortic lymph node metastases in pancreatic cancer should not be considered a watershed for curative resection. Sci Rep. 2017;7(1):7688.

8. Williams JL, Chan CK, Toste PA, Elliott IA, Vasquez CR, Sunjaya DB, et al. Association of Histopathologic phenotype of periampullary adenocarcinomas with survival. JAMA surgery. 2017;152(1):82-8.

9. Welsch T, Bork U, Distler M, Weitz J. Top-down approach to the superior mesenteric artery and the mesopancreas during pancreatoduodenectomy for pancreatic cancer. Journal of surgical oncology. 2016;113(6):668-71.

10. Moher D, Liberati A, Tetzlaff J, Altman DG, Group P. Preferred reporting items for systematic reviews and meta-analyses: the PRISMA statement. J Clin Epidemiol. 2009;62(10):1006-12.

11. Yoshida T, Matsumoto T, Sasaki A, Shibata K, Aramaki M, Kitano S. Outcome of paraaortic node-positive pancreatic head and bile duct adenocarcinoma. Am J Surg. 2004;187(6):736-40.

12. Hafeez Bhatti AB, Dar FS, Sahaab E, Khan NY, Zia H, Rana A, et al. Survival advantage with para aortic lymphadenectomy in peri-ampullary cancer: a retrospective cohort study. Int J Surg. 2016;31:58-62.

13. Connor S, Bosonnet L, Ghaneh P, Alexakis N, Hartley M, Campbell F, et al. Survival of patients with periampullary carcinoma is predicted by lymph node 8a but not by lymph node 16b1 status. Br J Surg. 2004;91(12):1592-9.

14. Murakami Y, Uemura K, Sudo T, Hashimoto Y, Nakashima A, Kondo N, et al. Is Para-aortic lymph node metastasis a contraindication for radical resection in biliary carcinoma? World J Surg. 2011;35(5):1085-93.

15. Farid SG, Falk GA, Joyce D, Chalikonda S, Walsh RM, Smith AM, et al. Prognostic value of the lymph node ratio after resection of periampullary carcinomas. HPB. 2014;16(6):582-91. 
16. Shamseddine Al, Mukherji D, Melki C, Elias E, Eloubeidi M, Dimassi $H$, et al. Lymph node ratio is an independent prognostic factor after resection of periampullary malignancies: data from a tertiary referral center in the Middle East. Am J Clin Oncol. 2014;37(1):13-8.

17. Tol JA, Brosens LA, van Dieren S, van Gulik TM, Busch OR, Besselink MG, et al. Impact of lymph node ratio on survival in patients with pancreatic and periampullary cancer. Br J Surg. 2015;102(3):237-45.

18. Liu B, Heckler M, Heger U, Roth S, Klaiber U, Buchler MW, et al. Definition of an extended minimum level of lymphadenectomy in non-pancreatic periampullary cancer resections. HPB. 2018.

19. van Rijssen LB, Narwade P, van Huijgevoort NC, Tseng DS, van Santvoort HC, Molenaar IQ, et al. Prognostic value of lymph node metastases detected during surgical exploration for pancreatic or periampullary cancer: a systematic review and meta-analysis. HPB. 2016;18(7):559-66.

\section{Publisher's Note}

Springer Nature remains neutral with regard to jurisdictional claims in published maps and institutional affiliations.

Ready to submit your research? Choose BMC and benefit from:

- fast, convenient online submission

- thorough peer review by experienced researchers in your field

- rapid publication on acceptance

- support for research data, including large and complex data types

- gold Open Access which fosters wider collaboration and increased citations

- maximum visibility for your research: over $100 \mathrm{M}$ website views per year

At BMC, research is always in progress.

Learn more biomedcentral.com/submissions 